\title{
Examining the Utility of 30-day Readmission Rates and Hospital Profiling in the Veterans Health Administration
}

\author{
Charlie M Wray, DO, MS ${ }^{1,2 *}$; Marzieh Vali, MS³; Louise C Walter, MD,4; Lenny Lopez, MD, MPH, MDiv ${ }^{1,2}$; \\ Peter C Austin, MSc, PhD'5; Amy Byers, $\mathrm{PhD}^{1}$; Salomeh Keyhani, MD, MPH'1,6
}

\begin{abstract}
${ }^{1}$ Department of Medicine, University of California, San Francisco, California; '2Division of Hospital Medicine, San Francisco Veterans Affairs Medical Center, San Francisco, California; ${ }^{3}$ Northern California Institute for Research and Education, San Francisco Veterans Affairs Medical Center, San Francisco, California; ${ }^{4}$ Division of Geriatrics, San Francisco Veterans Affairs Medical Center, San Francisco, California; ${ }^{5}$ nstitute of Health Policy, Management and Evaluation, University of Toronto, Ontario, Canada; ${ }^{6}$ Division of General Internal Medicine, San Francisco Veterans Affairs Medical Center, San Francisco, California.
\end{abstract}

BACKGROUND: The Veterans Health Administration (VA) reports hospital-specific 30-day risk-standardized readmission rates (RSRRs) using CMS-derived models.

OBJECTIVE: The aim of this study was to examine and describe the interfacility variability of 30-day RSRRs for acute myocardial infarction (AMI), heart failure (HF), and pneumonia as a means to assess its utility for VA quality improvement and hospital comparison.

RESEARCH DESIGN: A retrospective analysis of VA and Medicare claims data using one-year (2012) and three-year (2010-2012) data given their use for quality improvement or for hospital comparison, respectively.

SUBJECTS: This study included 3,571 patients hospitalized for AMI at 56 hospitals, 10,609 patients hospitalized for HF at 102 hospitals, and 10,191 patients hospitalized for pneumonia at 106 hospitals.

MEASURES: Hospital-specific 30-day RSRRs for AMI, HF, and pneumonia hospitalizations were calculated using hierarchical generalized linear models.
RESULTS: Of 164 qualifying VA hospitals, 56 (34\%), 102 (62\%), and 106 (64\%) qualified for analysis based on CMS criteria for AMI, HF, and pneumonia cohorts, respectively. Using 2012 data, we found that two hospitals (2\%) had CHF RSRRs worse than the national average (+95\% Cl), whereas no hospital demonstrated worse-than-average risk-stratified readmission Rate (RSRR; $+95 \% \mathrm{Cl}$ ) for AMI or pneumonia. After increasing the number of facility admissions by combining three years of data, we found that four (range: 3.5\%-5.3\%) hospitals had RSRRs worse than the national average $(+95 \% \mathrm{Cl})$ for all three conditions.

CONCLUSIONS: The Centers for Medicare and Medicaid Services-derived 30-day readmission measure may not be a useful measure to distinguish VA interfacility performance or drive quality improvement given the low facility-level volume of such readmissions. Journal of Hospital Medicine 2019;14:266-271. Published online first February 20, 2019. (C) 2019 Society of Hospital Medicine
U sing methodology created by the Centers for Medicare \& Medicaid Services (CMS), the Department of Veterans Affairs (VA) calculates and reports hospital performance measures for several key conditions, including acute myocardial infarction (AMI), heart failure (HF), and pneumonia. ${ }^{1}$ These measures are designed to benchmark individual hospitals against how average hospitals perform when caring for a similar case-mix index. Because readmissions to the hospital within 30-days of discharge are common and costly, this metric has garnered extensive attention in recent years.

To summarize the 30 -day readmission metric, the VA utilizes

\footnotetext{
*Corresponding Author: Charlie M. Wray, DO, MS; E-mail: Charlie.Wray@ucsf. edu; Telephone: 415-595-9662; Twitter: @WrayCharles

Additional Supporting Information may be found in the online version of this article.
}

Received: November 6, 2018; Revised: December 18, 2018;

Accepted: December 20, 2018

๑) 2019 Society of Hospital Medicine DOI 10.12788/jhm.3155 the Strategic Analytics for Improvement and Learning (SAIL) system to present internally its findings to VA practitioners and leadership. ${ }^{2}$ The VA provides these data as a means to drive quality improvement and allow for comparison of individual hospitals' performance across measures throughout the VA healthcare system. Since 2010, the VA began using and publicly reporting the CMS-derived 30-day Risk-Stratified Readmission Rate (RSRR) on the Hospital Compare website. ${ }^{3}$ Similar to CMS, the VA uses three years of combined data so that patients, providers, and other stakeholders can compare individual hospitals' performance across these measures. ${ }^{1}$ In response to this, hospitals and healthcare organizations have implemented quality improvement and large-scale programmatic interventions in an attempt to improve quality around readmissions. ${ }^{4-6} \mathrm{~A}$ recent assessment on how hospitals within the Medicare fee-for-service program have responded to such reporting found large degrees of variability, with more than half of the participating institutions facing penalties due to greater-than-expected readmission rates. ${ }^{5}$ Although the VA utilizes the same CMS-derived model in its assessments and reporting, the variability and distri- 
bution around this metric are not publicly reported-thus making it difficult to ascertain how individual VA hospitals compare with one another. Without such information, individual facilities may not know how to benchmark the quality of their care to others, nor would the VA recognize which interventions addressing readmissions are working, and which are not. Although previous assessments of interinstitutional variance have been performed in Medicare populations, ${ }^{7}$ a focused analysis of such variance within the VA has yet to be performed.

In this study, we performed a multiyear assessment of the CMS-derived 30-day RSRR metric for AMI, HF, and pneumonia as a useful measure to drive VA quality improvement or distinguish VA facility performance based on its ability to detect interfacility variability.

\section{METHODS}

\section{Data Source}

We used VA administrative and Medicare claims data from 2010 to 2012. After identifying index hospitalizations to VA hospitals, we obtained patients' respective inpatient Medicare claims data from the Medicare Provider Analysis and Review (MedPAR) and Outpatient files. All Medicare records were linked to VA records via scrambled Social Security numbers and were provided by the VA Information Resource Center. This study was approved by the San Francisco VA Medical Center Institutional Review Board.

\section{Study Sample}

Our cohort consisted of hospitalized VA beneficiary and Medicare fee-for-service patients who were aged $\geq 65$ years and admitted to and discharged from a VA acute care center with a primary discharge diagnosis of $\mathrm{AMI}, \mathrm{HF}$, or pneumonia. These comorbidities were chosen as they are publicly reported and frequently used for interfacility comparisons. Because studies have found that inclusion of secondary payer data (ie, CMS data) may affect hospital-profiling outcomes, we included Medicare data on all available patients. ${ }^{8}$ We excluded hospitalizations that resulted in a transfer to another acute care facility and those admitted to observation status at their index admission. To ensure a full year of data for risk adjustment, beneficiaries were included only if they were enrolled in Medicare for 12 months prior to and including the date of the index admission.

Index hospitalizations were first identified using VA-only inpatient data similar to methods outlined by the CMS and endorsed by the National Quality Forum for Hospital Profiling. ${ }^{9}$ An index hospitalization was defined as an acute inpatient discharge between 2010 and 2012 in which the principal diagnosis was AMI, HF, or pneumonia. We excluded in-hospital deaths, discharges against medical advice, and--for the AMI cohort only--discharges on the same day as admission. Patients may have multiple admissions per year, but only admissions after 30 days of discharge from an index admission were eligible to be included as an additional index admission.

\section{Outcomes}

A readmission was defined as any unplanned rehospitalization to either non-VA or VA acute care facilities for any cause within 30 days of discharge from the index hospitalization. Readmissions to observation status or nonacute or rehabilitation units, such as skilled nursing facilities, were not included. Planned readmissions for elective procedures, such as elective chemotherapy and revascularization following an AMI index admission, were not considered as an outcome event.

\section{Risk Standardization for 30-day Readmission}

Using approaches developed by CMS, ${ }^{10-12}$ we calculated hospital-specific 30-day RSRRs for each VA. Briefly, the RSRR is a ratio of the number of predicted readmissions within 30 days of discharge to the expected number of readmissions within 30 days of hospital discharge, multiplied by the national unadjusted 30-day readmission rate. This measure calculates hospital-specific RSRRs using hierarchical logistic regression models, which account for clustering of patients within hospitals and risk-adjusting for differences in case-mix, during the assessed time periods. ${ }^{13}$ This approach simultaneously models two levels (patient and hospital) to account for the variance in patient outcomes within and between hospitals. ${ }^{14}$ At the patient level, the model uses the log odds of readmissions as the dependent variable and age and selected comorbidities as the independent variables. The second level models the hospital-specific intercepts. According to CMS guidelines, the analysis was limited to facilities with at least 25 patient admissions annually for each condition. All readmissions were attributed to the hospital that initially discharged the patient to a nonacute setting.

\section{Analysis}

We examined and reported the distribution of patient and clinical characteristics at the hospital level. For each condition, we determined the number of hospitals that had a sufficient number of admissions $(n \geq 25)$ to be included in the analyses. We calculated the mean, median, and interquartile range for the observed unadjusted readmission rates across all included hospitals.

Similar to methods used by CMS, we used one year of data in the VA to assess hospital quality and variation in facility performance. First, we calculated the 30-day RSRRs using one year (2012) of data. To assess how variability changed with higher facility volume (ie, more years included in the analysis), we also calculated the 30-day RSRRs using two and three years of data. For this, we identified and quantified the number of hospitals whose RSRRs were calculated as being above or below the national VA average (mean $\pm 95 \% \mathrm{Cl}$ ). Specifically, we calculated the number and percentage of hospitals that were classified as either above $(+95 \% \mathrm{Cl})$ or below the national average $(-95 \% \mathrm{Cl})$ using data from all three time periods. All analyses were conducted using SAS Enterprise Guide, Version 7.1. The SAS statistical packages made available by the CMS Measure Team were used to calculate RSRRs.

\section{RESULTS}

\section{Patient Characteristics}

Patients were predominantly older males (98.3\%). Among those hospitalized for AMI, most of them had a history of 
previous coronary artery bypass graft (CABG) (69.1\%), acute coronary syndrome (ACS; 66.2\%), or documented coronary atherosclerosis (89.8\%). Similarly, patients admitted for HF had high rates of CABG (71.3\%) and HF (94.6\%), in addition to cardiac arrhythmias (69.3\%) and diabetes (60.8\%). Patients admitted with a diagnosis of pneumonia had high rates of CABG (61.9\%), chronic obstructive pulmonary disease (COPD; $58.1 \%)$, and previous diagnosis of pneumonia (78.8\%; Table 1). Patient characteristics for two and three years of data are presented in Supplementary Table 1.

\section{VA Hospitals with Sufficient Volume to Be Included in Profiling Assessments}

There were 146 acute-care hospitals in the VA. In 2012, 56 (38\%) VA hospitals had at least 25 admissions for AMI, 102 (70\%) hospitals had at least 25 admissions for CHF, and 106 (73\%) hospitals had at least 25 admissions for pneumonia (Table 1) and therefore qualified for analysis based on CMS criteria for 30-day RSRR calculation. The study sample included 3,571 patients with AMI, 10,609 patients with CHF, and 10,191 patients with pneumonia.

\section{0-Day Readmission Rates}

The mean observed readmission rates in 2012 were 20\% (95\% $\mathrm{Cl} 19 \%-21 \%)$ among patients admitted for AMI, 20\% $195 \% \mathrm{Cl}$ $19 \%-20 \%)$ for patients admitted with $\mathrm{CHF}$, and $15 \%(95 \% \mathrm{Cl}$ $15 \%-16 \%)$ for patients admitted with pneumonia. No significant variation from these rates was noted following risk standardization across hospitals (Table 2). Observed and risk-standardized rates were also calculated for two and three years of data (Supplementary Table 2) but were not found to be grossly different when utilizing a single year of data.

In 2012, two hospitals (2\%) exhibited HF RSRRs worse than the national average $(+95 \% \mathrm{Cl})$, whereas no hospital demonstrated worse-than-average rates $(+95 \% \mathrm{Cl})$ for $\mathrm{AMl}$ or pneumonia (Table 3, Figure 1). Similarly, in 2012, only three hospitals had RSRRs better than the national average $(-95 \% \mathrm{Cl})$ for HF and pneumonia.

We combined data from three years to increase the volume of admissions per hospital. Even after combining three years of data across all three conditions, only four hospitals (range: $3.5 \%-5.3 \%)$ had RSRRs worse than the national average (+95\% $\mathrm{Cl})$. However, four (5.3\%), eight (7.1\%), and 11 (9.7\%) VA hospitals had RSRRs better than the national average $(-95 \% \mathrm{Cl})$.

\section{DISCUSSION}

We found that the CMS-derived 30-day risk-stratified readmission metric for $\mathrm{AMI}, \mathrm{HF}$, and pneumonia showed little variation among VA hospitals. The lack of institutional 30-day readmission volume appears to be a fundamental limitation that subsequently requires multiple years of data to make this metric clinically meaningful. As the largest integrated healthcare system in the United States, the VA relies upon and makes large-scale programmatic decisions based on such performance data. The inability to detect meaningful interhospital variation in a timely manner suggests that the CMS-derived 30-day RSRR may not be a sensitive metric to distinguish facility performance or drive quality improvement initiatives within the VA.

First, we found it notable that among the $146 \mathrm{VA}$ medical centers available for analysis, ${ }^{15}$ between $38 \%$ and $77 \%$ of hospitals qualified for evaluation when using CMS-based participation criteria-which excludes institutions with fewer than 25 episodes per year. Although this low degree of qualification for profiling was most dramatic when using one year of data (range: 38\%-72\%), we noted that it did not dramatically improve when we combined three years of data (range: $52 \%$ $77 \%)$. These findings act to highlight the population and systems differences between CMS and VA populations ${ }^{16}$ and further support the idea that CMS-derived models may not be optimized for use in the VA healthcare system.

Our findings are particularly relevant within the VA given the quarterly rate with which these data are reported within the VA SAIL scorecard. ${ }^{2}$ The VA designed SAIL for internal benchmarking to spotlight successful strategies of top performing institutions and promote high-quality, value-based care. Using one year of data, the minimum required to utilize CMS models, showed that quarterly feedback (ie, three months of data) may not be informative or useful given that few hospitals are able to differentiate themselves from the mean ( $\pm 95 \% \mathrm{Cl}$ ). Although the capacity to distinguish between high and low performers does improve by combining hospital admissions over three years, this is not a reasonable timeline for institutions to wait for quality comparisons. Furthermore, although the VA does present its data on CMS's Hospital Compare website using three years of combined data, the variability and distribution of such results are not supplied. ${ }^{3}$

This lack of discriminability raises concerns about the ability to compare hospital performance between low- and high-volume institutions. Although these models function well in CMS settings with large patient volumes in which greater variability exists, ${ }^{5}$ they lose their capacity to discriminate when applied to low-volume settings such as the VA. Given that several hospitals in the US are small community hospitals with low patient volumes, ${ }^{17}$ this issue probably occurs in other non-VA settings. Although our study focuses on the VA, others have been able to compare VA and non-VA settings' variation and distribution. For example, Nuti et al. explored the differences in 30-day RSRRs among hospitalized patients with AMI, HF, and pneumonia and similarly showed little variation, narrow distributions, and few outliers in the VA setting compared to those in the non-VA setting. For small patient volume institutions, including the VA, a focus on high-volume services, outcomes, and measures (ie, blood pressure control, medication reconciliation, etc.) may offer more discriminability between high- and low-performing facilities. For example, Patel et al. found that VA process measures in patients with HF (ie, beta-blocker and ACE-inhibitor use) can be used as valid quality measures as they exhibited consistent reliability over time and validity with adjusted mortality rates, whereas the 30 -day RSRR did not. ${ }^{18}$

Our findings may have substantial financial, resource, and policy implications. Automatically developing and reporting measures created for the Medicare program in the VA may not 
TABLE 1. Characteristics of Patients Hospitalized for Acute Myocardial Infarction, Heart Failure, and Pneumonia

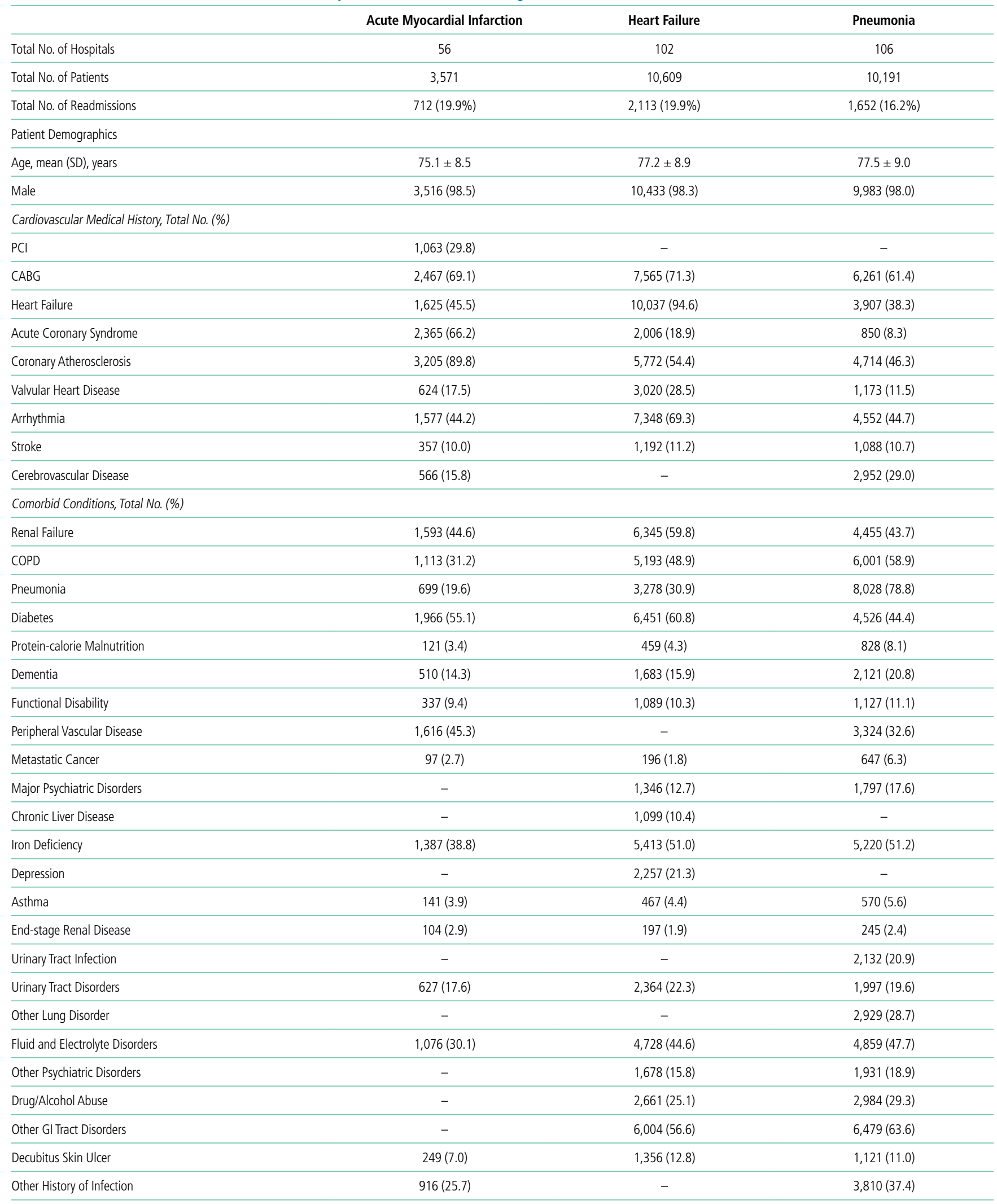

Note: Data calculated using 1-year of data (2012). CABG, coronary artery bypass graft; COPD, chronic obstructive pulmonary disease, GI, gastrointestinal; MI, myocardial infarction; PCl, percutaneous coronary intervention; VA, Veterans Affairs. Blank spaces (--) indicate that data were not included in risk adjustment of these publicly reported measures for a given condition or outcome. The following conditions were also controlled for but were not included in the above table: Anterior MI, Inferior, Lateral, or Posterior MI, Cardiopulmonary-respiratory failure and shock, nephritis, peptic ulcer, and other specified GI tract disorders, vertebral fractures, other injuries, septicemia, pleural effusion/pneumothorax, fibrosis of lung or other chronic lung disorders, other unspeci-

fied heart diseases, and severe hematologic disorders. 
TABLE 2. Distribution of 30-day Observed and Risk-Standardized Readmission Rates for VA Hospitals

\begin{tabular}{|c|c|c|c|c|c|c|}
\hline & \multicolumn{3}{|c|}{ Observed Readmission Rate } & \multicolumn{3}{|c|}{ Risk-Standardized Readmission Rate } \\
\hline & $\begin{array}{l}\text { Mean } \\
(95 \% \mathrm{Cl})\end{array}$ & $\begin{array}{c}\text { Median } \\
\left(25^{\text {th }}, 75^{\text {th }} \text { percentile }\right)\end{array}$ & Range & $\begin{array}{l}\text { Mean } \\
(95 \% \mathrm{Cl})\end{array}$ & $\begin{array}{c}\text { Median } \\
\left(25^{\text {th }}, 75^{\text {th }} \text { percentile }\right)\end{array}$ & Range \\
\hline Acute Myocardial Infarction & $\begin{array}{c}0.20 \\
(0.19,0.21)\end{array}$ & $\begin{array}{c}0.20 \\
(0.15,0.24)\end{array}$ & $0.09-0.35$ & $\begin{array}{c}0.20 \\
(0.19,0.21)\end{array}$ & $\begin{array}{c}0.20 \\
(0.19,0.21)\end{array}$ & $0.18-0.22$ \\
\hline Heart Failure & $\begin{array}{c}0.20 \\
(0.19,0.20)\end{array}$ & $\begin{array}{c}0.19 \\
(0.17,0.23)\end{array}$ & $0.06-0.32$ & $\begin{array}{c}0.20 \\
(0.20,0.21)\end{array}$ & $\begin{array}{c}0.20 \\
(0.19,0.21)\end{array}$ & $0.18-0.22$ \\
\hline Pneumonia & $\begin{array}{c}0.15 \\
(0.15,0.16)\end{array}$ & $\begin{array}{c}0.15 \\
(0.12,0.19)\end{array}$ & $0-0.26$ & $\begin{array}{c}0.16 \\
(0.16,0.17)\end{array}$ & $\begin{array}{c}0.16 \\
(0.16,0.17)\end{array}$ & $0.15-0.19$ \\
\hline
\end{tabular}

NOTE: Data calculated using 1-year of data.

TABLE 3. Hospital Performance Outlier Identification

\begin{tabular}{|c|c|c|c|}
\hline Condition & $\begin{array}{l}\text { No. of Hospitals } \\
\text { Below National Average } \\
(-95 \% \mathrm{Cl}),(\%)\end{array}$ & $\begin{array}{l}\text { No. of Hospitals that Performed } \\
\text { at National Average } \\
( \pm 95 \% \text { Cl), }(\%)\end{array}$ & $\begin{array}{c}\text { No. of Hospitals } \\
\text { Above National Average } \\
(+95 \% \mathrm{Cl}),(\%)\end{array}$ \\
\hline \multicolumn{4}{|c|}{ Acute Myocardial Infarction (Total No. of Hospitals) } \\
\hline 1 year (56) & $0(0)$ & $56(100)$ & $0(0)$ \\
\hline 2 years $(71)$ & $2(2.8)$ & $67(94.4)$ & $2(2.8)$ \\
\hline 3 years $(76)$ & $4(5.3)$ & $68(89.5)$ & $4(5.3)$ \\
\hline \multicolumn{4}{|c|}{ Heart Failure (Total No. of Hospitals) } \\
\hline 1 year (102) & $1(0.9)$ & $99(97.1)$ & $2(2.0)$ \\
\hline 2 years $(110)$ & $5(4.6)$ & $102(92.7)$ & $3(2.7)$ \\
\hline 3 years (112) & $8(7.1)$ & $100(89.3)$ & $4(3.6)$ \\
\hline \multicolumn{4}{|c|}{ Pneumonia (Total No. of Hospitals) } \\
\hline 1 year (106) & $2(1.9)$ & $104(98.1)$ & $0(0)$ \\
\hline 2 years (111) & $6(5.4)$ & $104(93.7)$ & $1(0.9)$ \\
\hline 3 years (113) & $11(9.7)$ & $98(86.7)$ & $4(3.5)$ \\
\hline
\end{tabular}

be a good use of VA resources. In addition, facilities may react to these reported outcomes and expend local resources and finances to implement interventions to improve on a performance outcome whose measure is statistically no different than the vast majority of its comparators. Such events have been highlighted in the public media and have pointed to the fact that small changes in quality, or statistical errors themselves, can have large ramifications within the VA's hospital rating system. ${ }^{19}$

These findings may also add to the discussion on whether public reporting of health and quality outcomes improves patient care. Since the CMS began public reporting on RSRRs in 2009, these rates have fallen for all three examined conditions (AMI, HF, and pneumonia), ${ }^{7,20,21}$ in addition to several other health outcomes. ${ }^{17}$ Although recent studies have suggested that these decreased rates have been driven by the CMS-sponsored Hospital Readmissions Reduction Program (HRRP), ${ }^{22}$ others have suggested that these findings are consistent with ongoing secular trends toward decreased readmissions and may not be completely explained by public reporting alone. ${ }^{23}$ Moreover, prior work has also found that readmissions may be strongly impacted by factors external to the hospital setting, such as patients' social demographics (ie, household income, social isolation), that are not currently captured in risk-prediction models. ${ }^{24}$ Given the small variability we see in our data, public reporting within the VA is probably not beneficial, as only a small number of facilities are outliers based on RSRR.

Our study has several limitations. First, although we adapted the CMS model to the VA, we did not include gender in the model because $>99 \%$ of all patient admissions were male. Second, we assessed only three medical conditions that were being tracked by both CMS and VA during this time period, and these outcomes may not be representative of other aspects of care and cannot be generalized to other medical conditions. Finally, more contemporary data could lead to differing results - though we note that no large-scale structural or policy changes addressing readmission rates have been implemented within the VA since our study period.

The results of this study suggest that the CMS-derived 30-day risk-stratified readmission metric for $\mathrm{AMI}, \mathrm{HF}$, and pneumonia may not have the capacity to properly detect interfacility variance and thus may not be an optimal quality indicator within the VA. As the VA and other healthcare systems continually strive to improve the quality of care they provide, they will require more accurate and timely metrics for which to index their performance. 

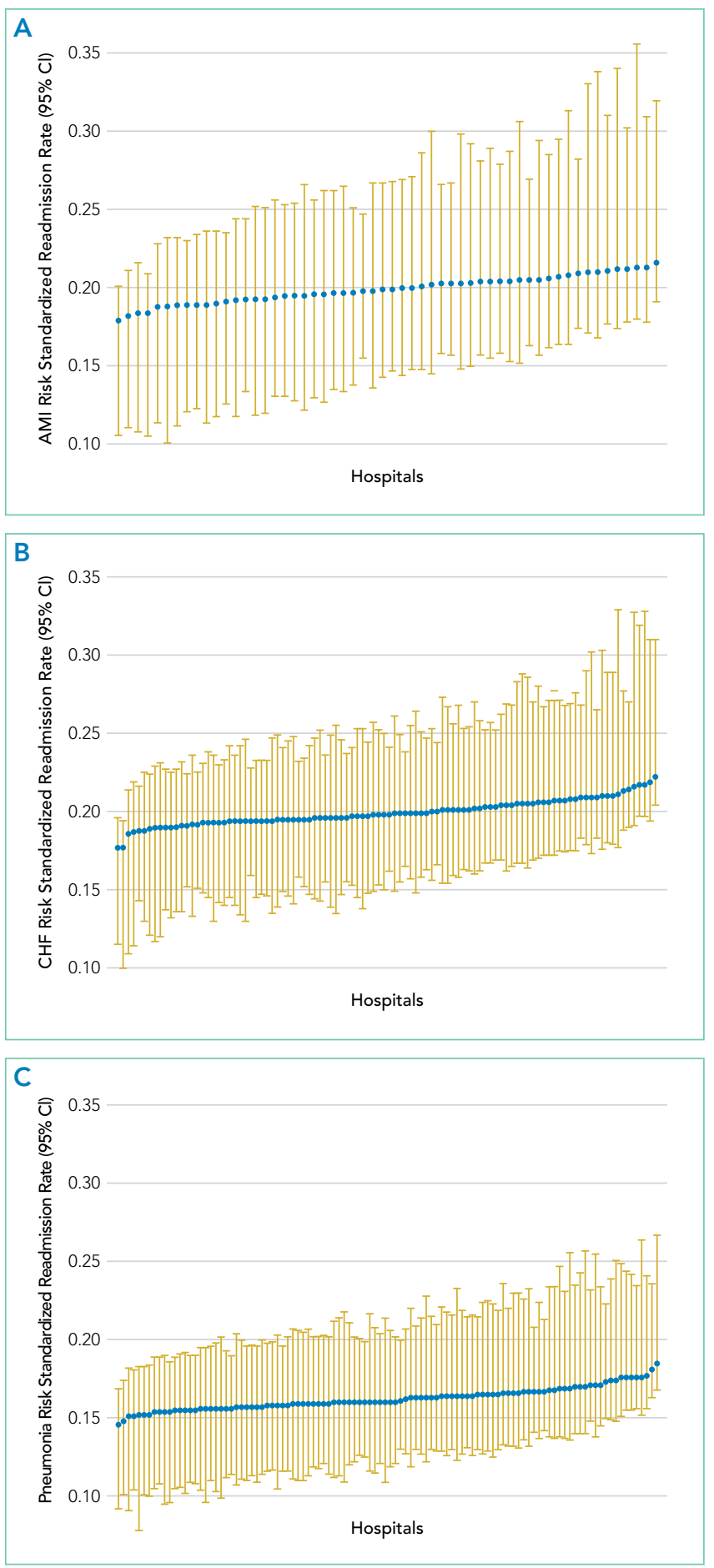

FIG 1. Hospital-Level Risk Standardized Readmissions Rates in VA Hospitals over One Year (2012). Point estimates with 95\% confidence intervals. Only hospitals with $>25$ admissions were included in the analysis. a) Acute myocardial infarction (AMI), b) Heart failure (HF), and c) Pneumonia.

Disclosures: The authors have nothing to disclose

\section{References}

1. Medicare C for, Baltimore MS 7500 SB, Usa M. VA Data. https://www.cms. gov/Medicare/Quality-Initiatives-Patient-Assessment-Instruments/HospitalQualitylnits/VA-Data.html. Published October 19, 2016. Accessed July 15, 2018.
2. Strategic Analytics for Improvement and Learning (SAIL) - Quality of Care. https://www.va.gov/QUALITYOFCARE/measure-up/Strategic_Analytics_ for_Improvement_and_Learning_SAIL.asp. Accessed July 15, 2018.

3. Snapshot. https://www.cms.gov/Medicare/Quality-Initiatives-Patient-Assessment-Instruments/HospitalQualitylnits/VA-Data.html. Accessed September 10, 2018.

4. Bradley $\mathrm{EH}$, Curry L, Horwitz LI, et al. Hospital strategies associated with 30day readmission rates for patients with heart failure. Circ Cardiovasc Qual Outcomes. 2013;6(4):444-450. doi: 10.1161/CIRCOUTCOMES.111.000101.

5. Desai NR, Ross JS, Kwon JY, et al. Association between hospital penalty status under the hospital readmission reduction program and readmission rates for target and nontarget conditions. JAMA. 2016;316(24):2647-2656. doi: 10.1001/jama.2016.18533.

6. Mcllvennan CK, Eapen ZJ, Allen LA. Hospital readmissions reduction program. Circulation. 2015;131(20):1796-1803. doi: 10.1161/CIRCULATIONAHA.114.010270.

7. Suter LG, Li S-X, Grady JN, et al. National patterns of risk-standardized mortality and readmission after hospitalization for acute myocardial infarction, heart failure, and pneumonia: update on publicly reported outcomes measures based on the 2013 release. J Gen Intern Med. 2014;29(10):1333-1340. doi: 10.1007/s11606-014-2862-5.

8. O'Brien WJ, Chen $\mathrm{Q}$, Mull HJ, et al. What is the value of adding Medicare data in estimating VA hospital readmission rates? Health Serv Res. 2015:50(1):40-57. doi: 10.1111/1475-6773.12207.

9. NQF: All-Cause Admissions and Readmissions 2015-2017 Technical Report. https://www.qualityforum.org/Publications/2017/04/All-Cause_Admissions_ and_Readmissions_2015-2017_Technical_Report.aspx. Accessed August 2, 2018

10. Keenan PS, Normand S-LT, Lin Z, et al. An administrative claims measure suitable for profiling hospital performance on the basis of 30-day all-cause readmission rates among patients with heart failure. Circ Cardiovasc Qual Outcomes. 2008:1(1):29-37. doi: 10.1161/CIRCOUTCOMES.108.802686.

11. Krumholz HM, Lin Z, Drye EE, et al. An administrative claims measure suitable for profiling hospital performance based on 30-day all-cause readmission rates among patients with acute myocardial infarction. Circ Cardiovasc Qual Outcomes. 2011;4(2):243-252. doi: 10.1161/CIRCOUTCOMES.110.957498.

12. Lindenauer PK, Normand S-LT, Drye EE, et al. Development, validation, and results of a measure of 30-day readmission following hospitalization for pneumonia. J Hosp Med. 2011;6(3):142-150. doi: 10.1002/jhm.890.

13. Medicare C for, Baltimore MS 7500 SB, Usa M. OutcomeMeasures. https:// www.cms.gov/Medicare/Quality-Initiatives-Patient-Assessment-Instruments/HospitalQualitylnits/OutcomeMeasures.html. Published October 13, 2017. Accessed July 19, 2018.

14. Nuti SV, Qin L, Rumsfeld JS, et al. Association of admission to Veterans Affairs hospitals vs non-Veterans Affairs hospitals with mortality and readmission rates among older hospitalized with acute myocardial infarction, heart failure, or pneumonia. JAMA. 2016;315(6):582-592. doi: 10.1001/ jama.2016.0278.

15. Solutions WW. Veterans Health Administration - Locations. https://www.va.gov/ directory/guide/division.asp?dnum=1. Accessed September 13, 2018.

16. Duan-Porter W (Denise), Martinson BC, Taylor B, et al. Evidence Review: Social Determinants of Health for Veterans. Washington (DC): Department of Veterans Affairs (US); 2017. http://www.ncbi.nlm.nih.gov/books/NBK488134/. Accessed June 13, 2018.

17. Fast Facts on U.S. Hospitals, 2018 | AHA. American Hospital Association. https://www.aha.org/statistics/fast-facts-us-hospitals. Accessed September 5, 2018.

18. Patel J, Sandhu A, Parizo J, Moayedi Y, Fonarow GC, Heidenreich PA. Validity of performance and outcome measures for heart failure. Circ Heart Fail. 2018;11(9):e005035.

19. Philipps D. Canceled Operations. Unsterile Tools. The V.A. Gave This Hospital 5 Stars. The New York Times. https://www.nytimes.com/2018/11/01/ us/veterans-hospitals-rating-system-star.html. Published November 3, 2018. Accessed November 19, 2018.

20. DeVore AD, Hammill BG, Hardy NC, Eapen ZJ, Peterson ED, Hernandez AF. Has public reporting of hospital readmission rates affected patient outcomes?: Analysis of Medicare claims data. J Am Coll Cardiol. 2016;67(8):963972. doi: 10.1016/j.jacc.2015.12.037.

21. Wasfy JH, Zigler CM, Choirat C, Wang Y, Dominici F, Yeh RW. Readmission rates after passage of the hospital readmissions reduction program: a prepost analysis. Ann Intern Med. 2017:166(5):324-331. doi: 10.7326/M16-0185.

22. Medicare C for, Baltimore MS 7500 SB, Usa M. Hospital Readmission Reduction Program. https://www.cms.gov/Medicare/Quality-Initiatives-Patient-Assessment-Instruments/Value-Based-Programs/HRRP/Hospital-Readmission-Reduction-Program.html. Published March 26, 2018. Accessed July 19, 2018.

23. Radford MJ. Does public reporting improve care? J Am Coll Cardiol. 2016;67(8):973-975. doi: 10.1016/j.jacc.2015.12.038.

24. Barnett ML, Hsu J, McWilliams JM. Patient characteristics and differences in hospital readmission rates. JAMA Intern Med. 2015;175(11):1803-1812. doi: 10.1001/jamainternmed.2015.4660. 